\title{
Scaling aspects of the sea-ice-drift dynamics and pack fracture
}

\author{
A. Chmel $^{1}$, V. N. Smirnov ${ }^{2}$, and L. V. Panov ${ }^{2}$ \\ ${ }^{1}$ Fracture Physics Department, Ioffe Physico-Technical Institute, Russian Academy of Sciences, 194021 St. Petersburg, \\ Russia \\ ${ }^{2}$ Arctic and Antarctic Research Institute, Bering street, 38, 199397 St. Petersburg, Russia
}

Received: 12 October 2006 - Published in Ocean Sci. Discuss.: 30 January 2007

Revised: 12 April 2007 - Accepted: 15 May 2007 - Published: 30 May 2007

\begin{abstract}
A study of the sea-ice dynamics in the periods of time prior to and during the cycles of basin-wide fragmentation of the ice cover in the Arctic Ocean is presented. The fractal geometry of the ice-sheets limited by leads and ridges was assessed using the satellite images, while the data on the correlated sea-ice motion were obtained in the research stations "North Pole 32" and "North Pole 33" established on the ice pack. The revealed decrease of the fractal dimension as a result of large-scale fragmentation is consistent with the localization of the fracture process (leads propagation). At the same time, the scaling properties of the distribution of amplitudes of ice-fields accelerations were insensitive to the event of sea-ice fragmentation. The temporal distribution of the accelerations was scale-invariant during "quiet" periods of sea-ice drift but disordered in the period of mechanical perturbation. The period of decorrelated (in time) ice-field motion during the important fracture event was interpreted as an inter-level transition in the hierarchic dynamical system. The mechanism of the long-range correlations in the sea-ice cover, including the fracture process, is suggested to be in relation with the self-organized oscillation dynamics inherent in the ice pack.
\end{abstract}

\section{Introduction}

Two continuous processes determine, mainly, the mechanical behavior of the ice pack in the Arctic Ocean: the sea-ice drift and pervasive fracturing. These processes are highly interrelated because the fracture events occur due to interaction between ice floes, and the large scale deformations are caused by the non-uniform pack motion. In recent years, it was revealed that the mechanical behavior of the Arctic sea-ice cover (ASIC) can be characterized by various scal-

Correspondence to: A. Chmel

(chmel@mail.ioffe.ru) ing exponents related to the elastic and strength properties of ice sheets. First of all, the plate-size distribution (number of ice pieces as a function of their dimension) follows the power law over several orders of magnitude (Matsushita, 1985; Korsnes et al., 2004) as well as the geometric proportions between the area and "coastline" of ice floes (Chmel et al., 2005). This suggests that the plate dimension distribution in the ASIC is fractal (Hopkins et al., 2004). The deformation rate that determines the fracture pattern in the ASIC is also scale invariant (Marsan et al., 2004). These spatial characteristics are combined with the temporal invariance of the sea ice motion (Chmel et al., 2005).

The mentioned above scaling features signalize that the mechanical behavior of the ice pack is highly correlated on scale levels, at least, 10 to $10^{3} \mathrm{~km}$ (Weiss, 2003; Marsan et al., 2004) (or, in terms of Overland (1995), from the level of individual plates to climate geophysical scales). This work is to demonstrate the response of the fractal properties of the lead-and-ridge pattern to basin-wide fracture events, and to consider the underlying mechanism of the correlated fracture in the ASIC issuing from the dynamics of pack motion. In other words, we show some intrinsic features of the seaice-drift dynamics and pack fracture seen by statistical physicists. The sources of information were the databases of field observations carried out in the ice-research stations of series "North Pole" (NP) NP 32 and NP 33 supplemented with the images from the NOAA satellite.

\section{Fractality}

\subsection{Guiding analogy}

In order to illustrate the scale invariance of the fracture events in nature, we shall consider the following example. Let us imagine a stone wall that bears a load or, at least, its own weight. The wall undergoes the action of wind pressure,

Published by Copernicus GmbH on behalf of the European Geosciences Union. 


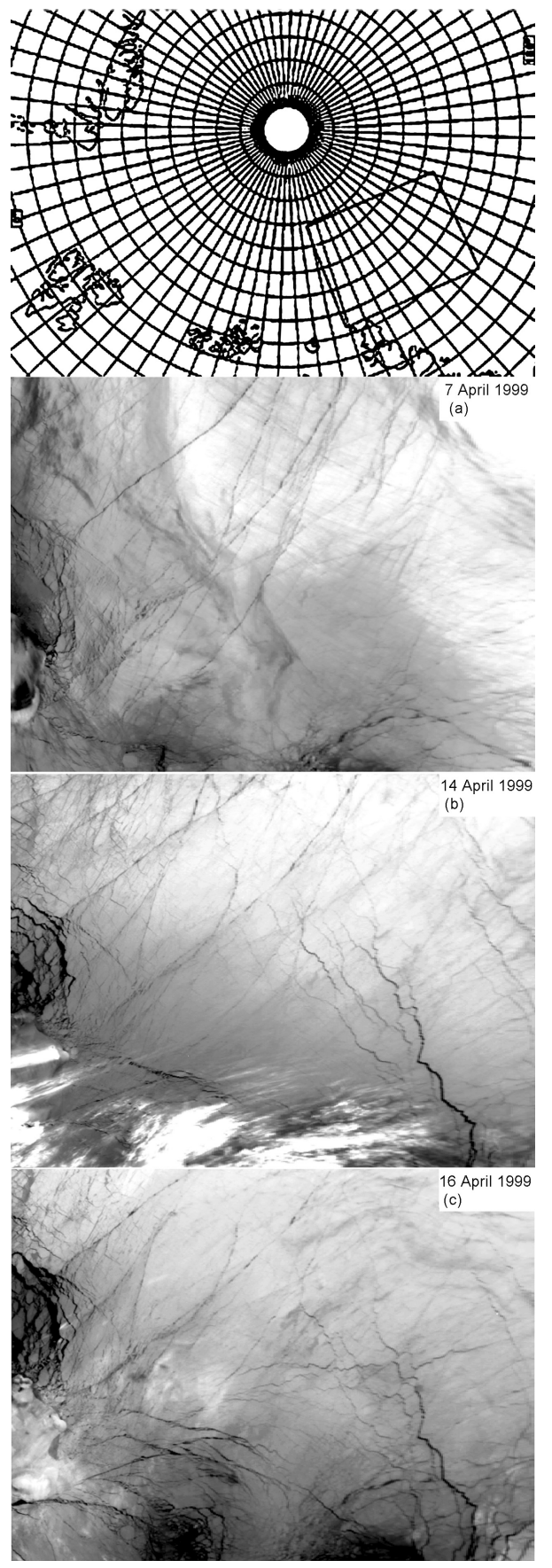

Fig. 1. A series of images dated of April 1999 when a giant lead nucleated and propagated over about $300 \mathrm{~km}$. The region covered by the image is shown on the top.

temperature gradients, solar radiation, etc. The aging processes develop themselves step by step. One day, or to be more precise, at a certain moment, the internal deformations overcome the aging-modified ultimate values, and the wall breaks down to pieces of different sizes. Numerous experiments and observations of fracture events of different kind

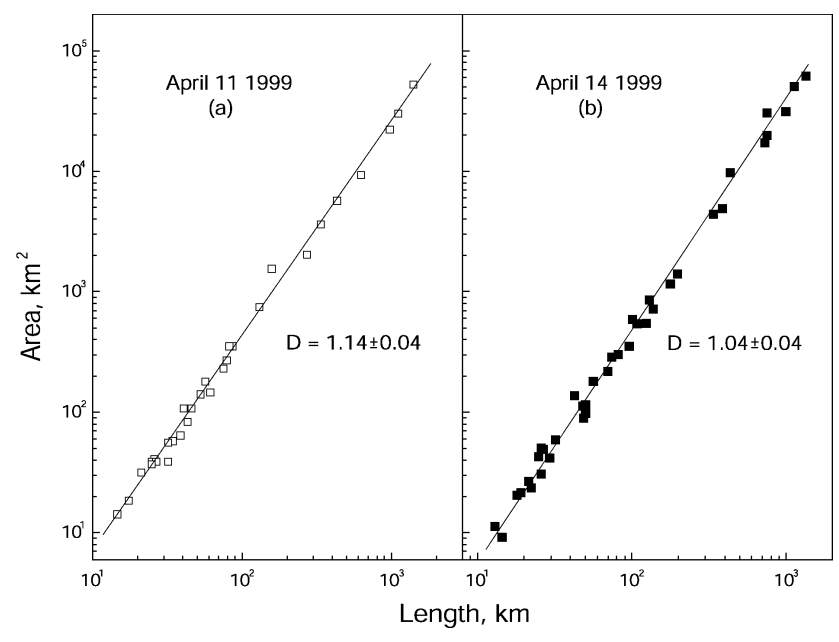

Fig. 2. Area versus perimeter dependences for ice-floes fragments resolved in images obtained prior to the large event (a), on the day of event (b) and a few weeks after the event (c). The straight lines fit the power law dependence (2). The shown values of $D$ were found from the slope of lines. The accuracy of the determination of D was estimated from the deviation of the slope at the end points of log-log plots with respect to the best-fit data of S versus $L$ dependences.

suggest that if one measures the size distribution of pieces, it would be turned out that the number of wall fragments $(n)$ is corresponded to their size $(r)$ by the power law:

$n(r) \propto r^{-D}$

where the power index $D$ is the fractal dimension of the ensemble of fracture products. The experiment similar to this mental illustration was carried out by Carpinteri et al. (2004) who studied the breakage of blocks of constructional materials and confirmed the validity of relation (1). The occurrence of the power-law statistics evidences the absence of characteristic lengths because the function $n(r)$ satisfies the scaling equation:

$n(\lambda r)=\lambda^{-D} n(r)$.

where $\lambda$ is the scale factor. In our example, this means that the number of wall fragments of different sizes changes with the scale of observation $\lambda r$ as given by the Eq. (1a). The power law distribution of fracture products results from the long-range correlation of the fracture process itself: each new breakage of links affects not only the neighboring sites but the whole ensemble of sites in a way to maintain the critical state (Caldarelli et al., 1996).

The ice cover is a kind of "horizontal" wall. Individual ice plates are restricted by leads (open water), cracks or pressure ridges. The action of compressive, tensile and shear stresses causes its fragmentation on various scale levels. Matsushita (1985) was the first who analyzed the size distribution of ice pieces (in the aerial photos of the frozen Okhotsk Sea) and he found the same relation (1) between the number and linear 
dimension of ice plates. Thus he had shown that the ice-cover fragmentation occur in a similar way on all scale levels.

The scale invariance of this kind can be characterized quantitatively be the value of fractal dimension, which is available, in particular, from measurements of the area $(S)$ and perimeter $(L)$ of fractal collection of two-dimensional objects; the relation between $S$ and $L$ is also expressed by the power law (Mandelbrot et al., 1984):

$S^{D} \propto L^{2}$.

In the next section we shall demonstrate the validity of the relation (2) as applied to the vast areas of the fragmented ice cover, and consider the response of the value $D$ to large scale mechanical perturbations in the ASIC.

\subsection{Sea-ice cover fragmentation}

\subsubsection{Shore interference}

Figure 1 shows a series of AVHRR images of April 1999 where a large-scale event was detected in the geographical region depicted in the cut-in. The appearance of the branching lead on 14 April was followed by prevailing transverse fragmentation with formation of smaller "debris" (compare Fig. $1 \mathrm{~b}$ and Fig. 1c). We suppose that this fracture event was caused by the shore interference that affected strongly the ice cover drift in April 1999. This was concluded from the monitoring of a set of reference points (well-recognizable patches of free water selected throughout the region of observation). From 7 to 12 April the distances between reference points gradually decreased so indicating the effect of ice compression. The quasi-stationary drift was disturbed on 12-13 April by the appearance of a strong rotational component accompanied with translations along the leads. Such the behavior is typical for ice-cover/offshore interaction. The measured dependences of $\mathrm{S}$ on $\mathrm{L}$ referred to images obtained prior to and after ice fracturing are depicted in Fig. 2. One can see a difference in the slope of two $\mathrm{L}$ versus $\mathrm{S}$ linear dependences plotted in doubly logarithmic coordinates. According to relation (2), the increase of the slope after the event of fragmentation signifies the decrease of the $D$-value (from $1.14 \pm 0.04$ to $1.04 \pm 0.04$ ). Physically, the decrease of $D$ evidences the localization of the process; in our case, this indicates a partial loss of the planar structure with a trend to one-dimensional organization (propagating leads). The fractal dimension was also determined from satellite images obtained a few days prior to 14 April 1999, and a few weeks after that day. Unfortunately, during the posterior period the cloudiness shaded the ice-cover pattern, and it was impossible to measure the geometric parameters of ice pieces. A variation of the fractal dimension value of the sea-ice cover pattern in the available period of time is shown in Fig. 3a.

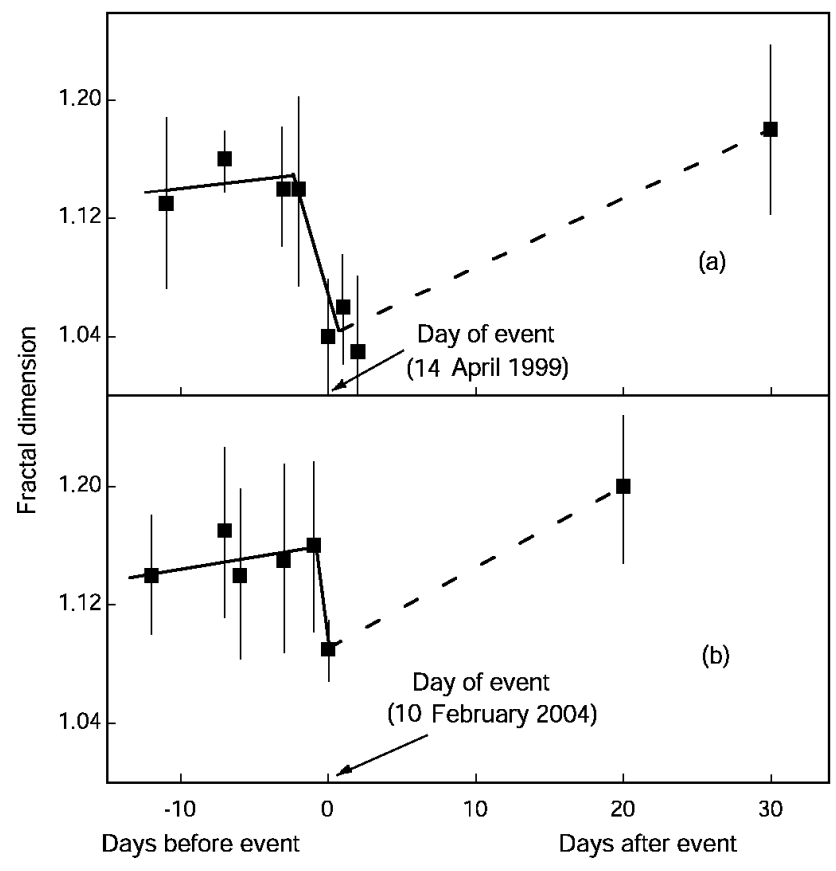

Fig. 3. Variation of the fractal dimension value of the sea-ice cover pattern in 1999 (a) and 2004 (b). The lines serve as guides for the eyes. The data in the periods of time after large events were not available due to the dense cloudiness (designated by dotted lines).

\subsubsection{Atmospheric depression}

The ice-drift in the Arctic Ocean is driven, mainly, by the wind forcing (Lewis et al., 1994; Richter-Menge and Elder, 1998), and the variability of the weather pattern causes cycles of redistribution of stresses and deformations with ice breaking (Hopkins et al., 2004) followed by refreezing (Korsnes et al., 2004). On 10 February 2004, a basin-wide seaice fragmentation occurred, and it was detected in the satellite images. Figure 4 shows the NOAA satellite images of the region of drift obtained on 9 February and 10 February 2004. In Fig. 4b one can see large-scale leads (up to $400 \mathrm{~km}$ in length), which formed in one day between two subsequent images and a position of the research station NP 32 that drifted on the pack during that period of time. The displacements and fragmentation of the ice-cover lasted during the whole February; on 2 March 2004 the research station NP 32 was abandoned in connection with multiple breakage of the ice-field on which the observations were carried out.

Any shore interference is not seen in the images; therefore, the large-scale perturbation was referred to a passage of the deep atmospheric depression over the region. This depression was recorded in the database of the station NP 32 (Chmel and Smirnov, 2005) in the time interval covering the event of 10 February 2004. The presence of the station NP 32 in the region of perturbation gave us a rare opportunity to put in comparison the visual data obtained using the remote technique with the results of field observations. 


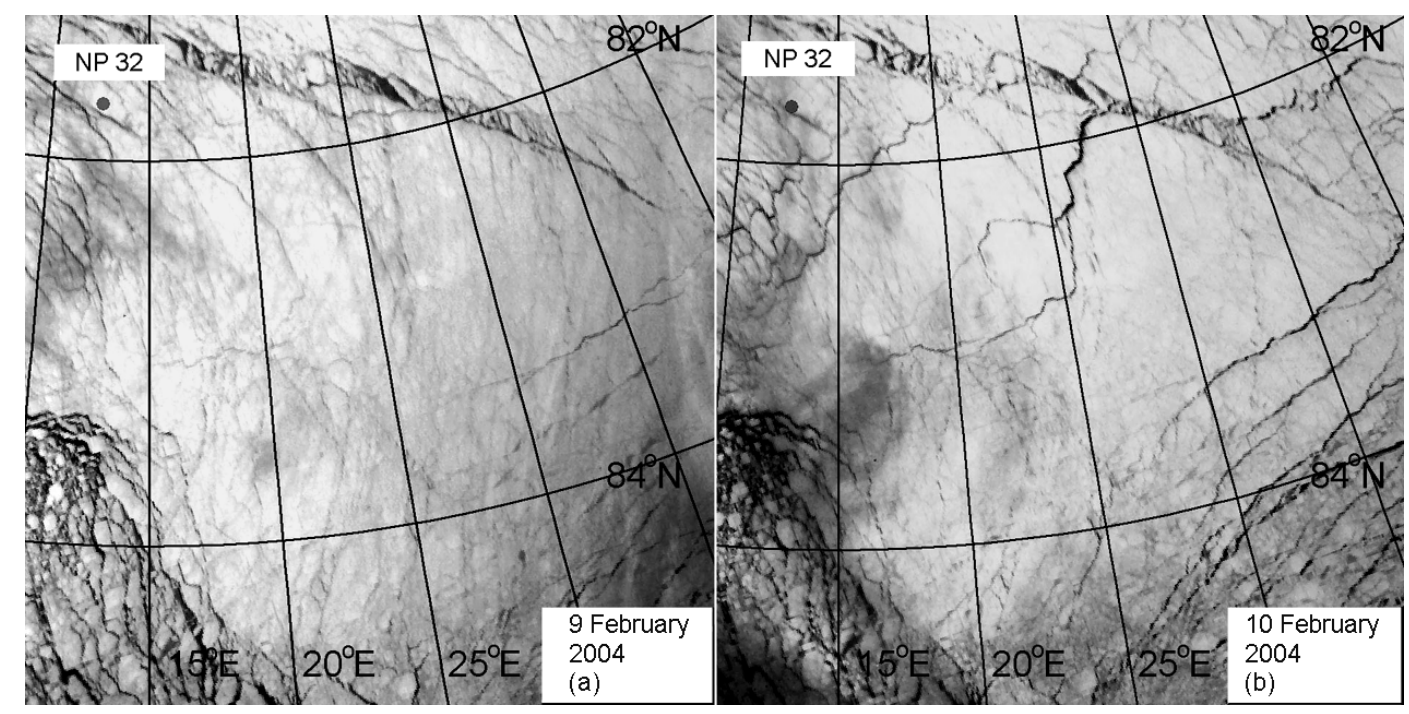

Fig. 4. $400 \times 400 \mathrm{~km}$ fragments of satellite images of the region of NP 32 drift obtained on 9 February (a) and 10 February (b) 2004. A branched, newly formed lead is seen in (b).

A variation of the $D$-value in the period of interest (Fig. 3b) was available from the analysis of a series of satellite images obtained before and after the catastrophic fragmentation. Similar to the event of 14 April 1999, a certain decrease of $D$ was detected in the day of event of 10 February 2004.

A posterior analysis of the database of field observations revealed a response to the event of 10 February not only in the geometric relations of ice floes, but also in the dynamics of sea-ice motion. In the next Section, the temporal correlation of ice-field accelerations in quiet and excited periods of the ice drift will be considered.

\section{Drift dynamics}

\subsection{Accelerations}

The drift of the station NP 32 was monitored with the help of the GPS system with space and time resolutions $15 \mathrm{~m}$ and $10 \mathrm{~min}$, respectively. On the base of these data, the ice-field speed $(V)$ and accelerations $A=\Delta V / \Delta t$ were calculated (here $\Delta V$ is the variation of drift rate measured in regular intervals $\Delta t=10 \mathrm{~min}$ ).

It has been shown previously (Chmel et al., 2005), that both positive and negative accelerations produce an approximately equivalent contribution to the total distribution of accelerations. Therefore, when processing the data, only the absolute values of accelerations were under consideration:

$A \equiv|A|=|\Delta V| / \Delta t$.

A sequence of ice-field accelerations recorded in the time interval covering the event of 10 February 2004 is depicted in Fig. 5.

\subsection{Temporal correlation}

A temporal correlation between events can be found from the time distribution of events of a certain importance. In this work, a time series of values A determined during JanuaryFebruary, 2004 was used to construct the function of distribution of intervals (waiting times, $\tau$ ) between the most significant accelerations, that is between those whose amplitudes exceed a certain cut-off value $A_{\text {cut-off }}: N_{A>\text { Acut-off }}(>\tau)$. To specify the value $A_{\text {cut-off }}$, one should take into account a well-established trend in correlated statistical series of different nature: the greater event the more correlation (Christensen and Olami, 1992). In this analysis we considered the contribution of accelerations with amplitudes

$A>A_{\text {cut }- \text { off }}=8 \times 10^{-4} \mathrm{~m} / \mathrm{s}^{2}$.

These accelerations constituted $\sim 20$ per cent of all drift-rate variations detected during the given period of time. A range of variation of times $\tau$ covered the range from $6 \times 10^{2} \mathrm{~s}$ to $\sim 1.3 \times 10^{4} \mathrm{~s}$.

The functions $N_{A>\text { Acut-off }}(>\tau)$ were found for three periods of observations: (a) from 17 January to 31 January (a "remote" period); (b) 1 February to 10 February (a period prior to the "catastrophic" ice perturbation); (c) 11 February to 28 February (a "posterior" period).

To construct $N_{A>\text { Acut-off }}(>\tau)$ graphically, the number of subsequent events $N_{A>\text { Acut-off }}$ separated by the time interval that exceeds $\tau$ was plotted against $\tau$ in doubly logarithmic coordinates (Fig. 6). Three constructed distributions of waiting time are unambiguously different. In Figs. 6a and c, corresponded to remote and posterior periods, respectively, the $N_{A}$ versus $\tau$ dependences exhibit the power law:

$N_{A>A \text { cut }- \text { off }}(>\tau) \propto \tau^{\delta}$ 
shown by straight lines in these figures. The power law distribution of waiting times is an indication of the long-time correlation between events: "the system refers to its history in order to define its future" (Kapiris et al., 2003). At the same time, the time sequence of ice-field accelerations does not follow the power law in the immediate time interval before the "catastrophic" perturbation (Fig. 6b).

The latter finding evidences the decorrelation of ice-drift dynamics in the pre-catastrophic period of time. To understand the mechanism of this decorrelation, we analyzed the field records of meteorological parameters and found that the period 1 to 10 February was characterized by very low air temperatures $\left(-35\right.$ to $\left.-45^{\circ} \mathrm{C}\right)$. We suppose that such temperature drops induce freezing cycles with suppressing the mobility of ice sheets, and, thus, disturb the temporal correlation of the drift. In addition, a restricted motion of ice-sheets hinders both the energy exchange, and the stress/deformation redistribution. During prolonged freezing cycles, such as that that occurred in February 2004, the suppression of the energy discharge through local shear and impact interactions caused the appearance of zones of overstress and promoted the ice cover fragmentation.

\subsection{Force scaling}

The distribution of forces acting on the ice-field of a given mass is identical to the distribution of its accelerations. Therefore, in order to determine the distribution of local forces that induced the accelerations, the amplitude distribution functions $N(>A)$ (here $N$ is the number of events, in which the amplitude of acceleration exceeds A) were constructed using the January and February databases of the NP 32. The functions plotted in log-log coordinates (Fig. 7) exhibit portions approximated with straight lines over at least one and a half decade. The exponents of the power law:

$N(>A) \propto A^{-\gamma}$

were found from the graphs' slope. Unlike the exponent $\tau$ that characterizes the temporal pattern of accelerations, the values $\gamma$ were found to be close to each other in January and February (3.0 \pm 0.1 and $3.2 \pm 0.3$, respectively). The dividing of the February database into two portions related with "catastrophic" and "posterior" periods did not affect the result (not shown) but made the statistics worse. Consequently, the force distribution in the sea-ice remains scale-invariant both in quiet and excited periods of the drift.

\section{Oscillation processes}

The above described scale invariance of the fragmentation and drift of the sea ice was formally derived from either spatial or temporal relations between the parameters characterizing, respectively, the geometry and the motion of the ice cover. The shown satellite images give a picture of

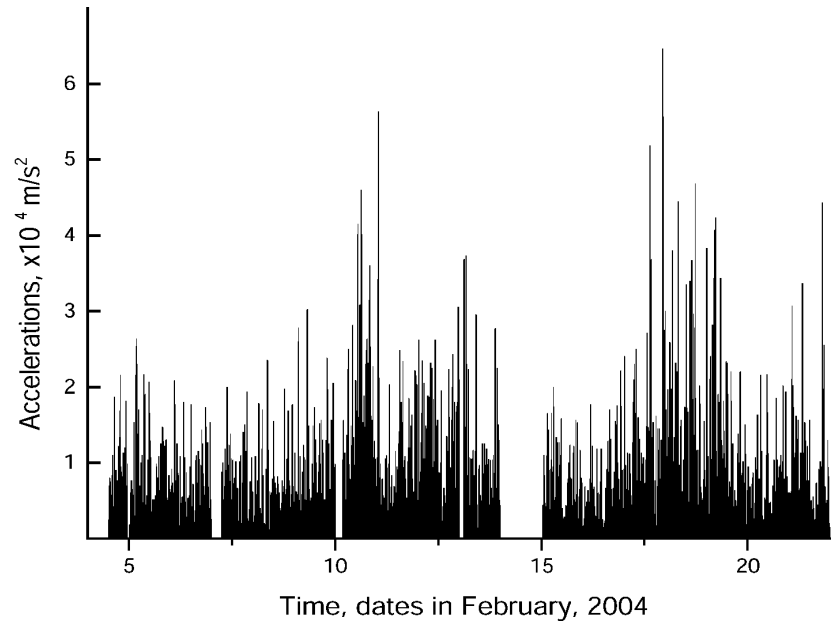

Fig. 5. Amplitudes of accelerations measured in the time interval from 4 February to 22 February 2004. A gap on 14-15 February is caused by excluding the data recorded during the ice breakage in the close vicinity of the GPS sensor position.

the self-organized behavior of the sea-ice both during the quasi-stationary drift, and in the periods of mechanical perturbations. Meanwhile, the mechanism, which controls the long-range interaction of sea-ice cover fragments and the "memory" about preceding events (expressed by the power law distributions of, respectively, dimensions and times), remains shaded since the driving force of the self-organization cannot be determined in the framework of the statistical physics (excluding general thermodynamical principles, $\mathrm{Ar}$ neodo, 1995). A formalistic description of events restricts the possibility of the analysis and prediction of the behavior of sea ice even with taking into account the features of self-organization. Therefore, one should search the physical processes in the ice pack, which could result in correlated fracture events in the ASIC. In this light, the pack oscillations are of particular interest since the oscillation processes of various nature arise and extend in the sea ice permanently thus maintaining the dynamical connectedness between components and realizing long-range interactions over this dynamical system (Smirnov, 1995, and references therein).

The spectrum of oscillations is composed of periodic motions due to ripple and those due to slip-stick and impact interactions accompanied with hummocking and cracking. In contact interactions the amplitudes of the horizontal displacements are much higher than the amplitudes of the vertical components (Martin and Drucer, 1991). The character of elastic waves is directly connected with the value and distribution of the deformations and stresses.

Figure 8a represents a typical example of horizontal oscillations excited by shearings, which were recorded with the help of accelerometers in the station NP 33 in June, 2005. Chaotic oscillations at $0.8 \mathrm{~Hz}$ (Fig. 8b) transformed to quasi-harmonic auto-oscillations; in a few minutes the 


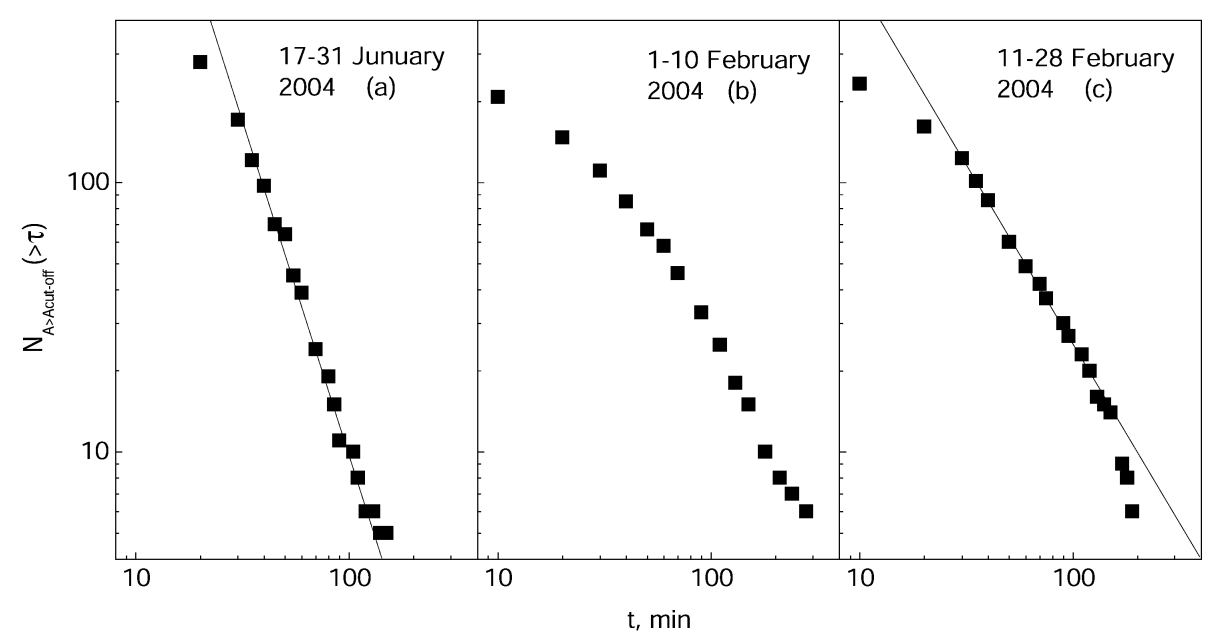

Fig. 6. Number of waiting times $N_{A}(>\tau)$ with duration higher than given by the corresponding abscissa. The straight lines fit the power law $N_{A}(>\tau) \propto \tau^{\gamma}$. Only accelerations with amplitudes higher than $A_{\text {cut }- \text { off }}=8 \times 10^{-4} \mathrm{~m} / \mathrm{s}^{2}$ were taken into account.

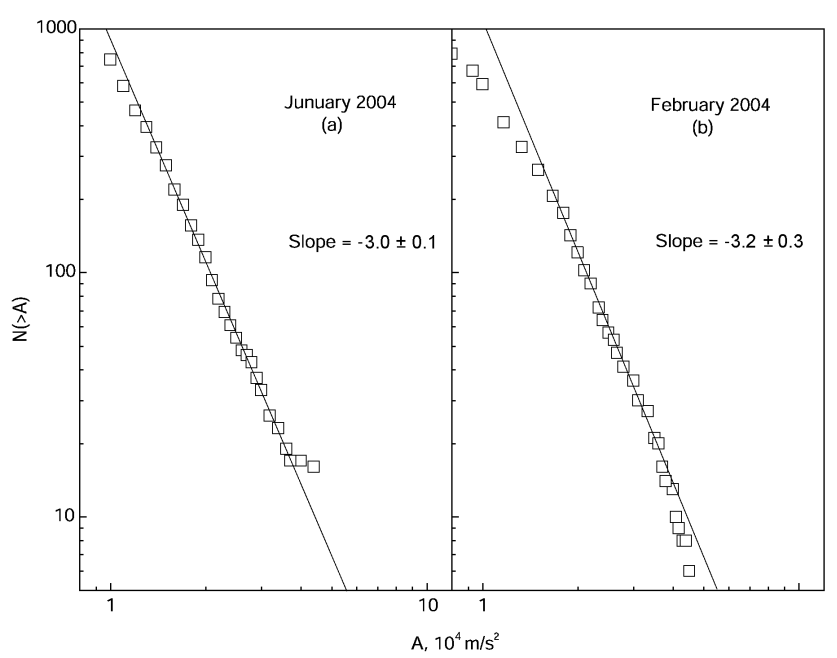

Fig. 7. Number of accelerations with amplitudes higher than that given by the corresponding abscissa. The straight lines show the power law $N(>A) \propto A^{-\delta}$.

process ceased due to aligning of the interacting components. No relations with meteo-factors were detected in this and other similar records; consequently, the observed autooscillations emerge as a self-organizing process under the permanent (non-periodic) external force. Synchronous observations carried out using sensors established on adjacent ice fields showed that the correlation radius of such phenomena could exceed the dimensions of individual pack components with involving into the correlated motion important areas up to the basin scale (Smirnov, 1995).

The oscillation processes in the sea ice are highly affected by the rheological properties of this medium. Under the prolonged (non-impact) action of the compressive/tensile force, a feedback between the energy input and the deformation properties of the oscillating domain composed of adjacent sheets takes place; this effect causes the parametric redistributing of the vibrational energy. As a result, the period and amplitude of oscillations do not depend on the outer forcing and are determined by the properties of the vibrational system. The parametric interaction leads to the autooscillations seen in Fig. 8. In contrast to forced oscillations under the action of periodic force, the energy input from a non-vibrational external source to the auto-oscillation dissipative system is dosed by the system itself. Thus, the energy of compression/tension transforms to the correlated motion of ice sheets.

\section{Discussion}

The scaling properties of the sea-ice drift dynamics and fracturing in the ASIC are shown to be sensitive to largescale perturbations. The fractal dimension of the collection of fragments limited by leads, cracks and ridges decreases in response to the fracture events in agreement with well-established trend of decreasing the dimensionality of the fracturing system due to fault nucleation (Zang, 1998; Kapiris 2004). (A term "fault" in this context is applicable to giant leads like those seen in Fig. 1b and Figs. 4b, c, Weiss, 2003).

The response of scale-invariant parameters of the drift dynamics is not so well defined. The distribution of the amplitudes of accelerations, which is equivalent to the force distribution in the ice cover, was found to be almost the same both in the period of quasi-stationary drift and during the basinwide ice cover fragmentation. The temporal correlation (expressed by the power-law distribution of waiting times between significant accelerations in the quiet period of drift) 

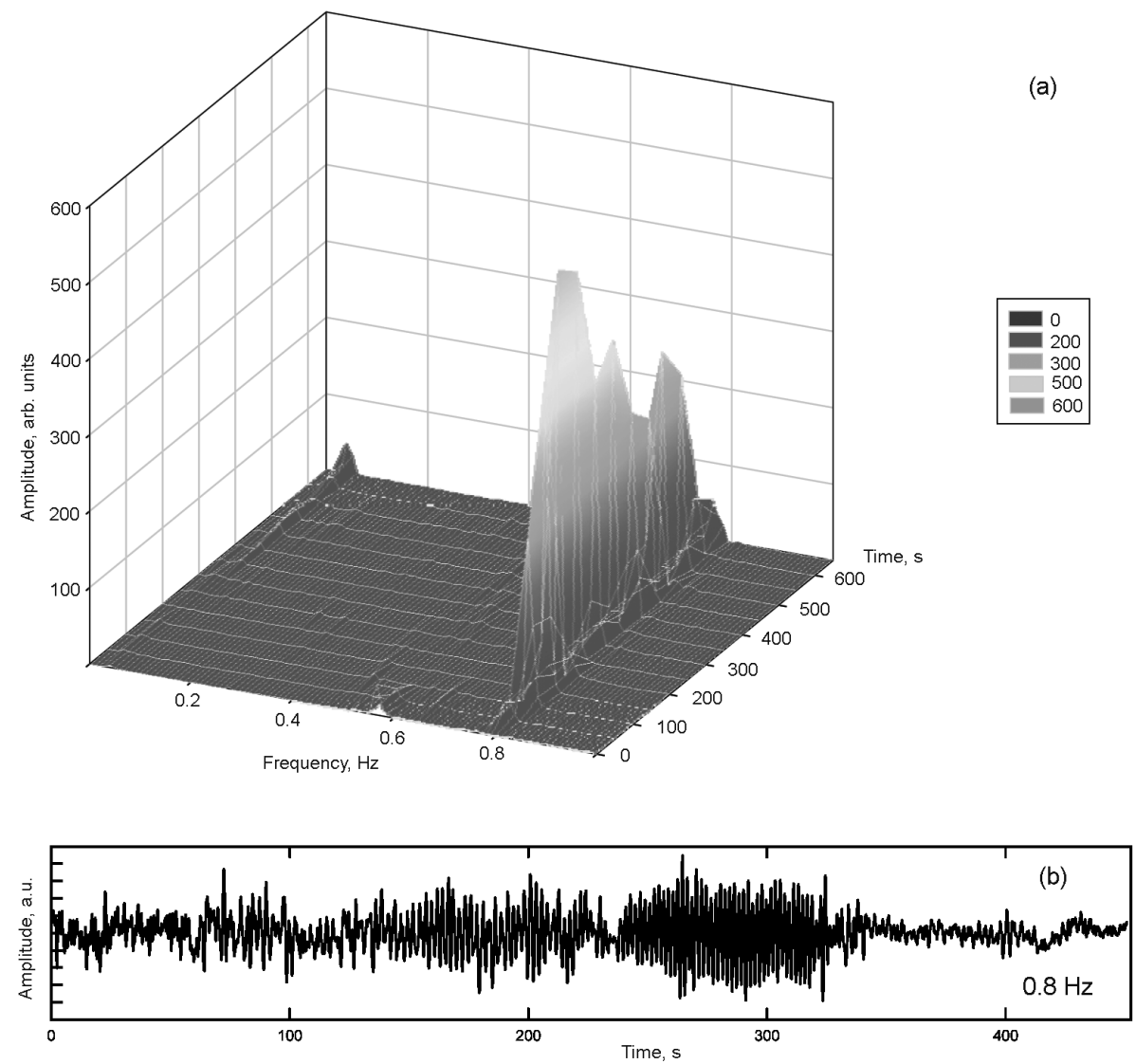

Fig. 8. Spectrum of oscillations induced by horizontal displacements of the ice-field recorded in the NP 33 in June 2005 (a). (b) shows the formation and frustration of quasi-harmonic oscillations at frequency $0.8 \mathrm{~Hz}$.

was, in contrast, disturbed during the intensive fracture process.

Scaling is inherent to critical phenomena where an insignificant event could trigger a "global" perturbation. The lack of the time correlation in the behavior of a critical system occurs, for example, under the condition of phase transition when the temporal sequence of events is fully disordered. In this light, the fracture of heterogeneous materials could be regarded as a sort of dynamic phase transition (Bouchaud, 1997). The used term "heterogeneous" implies the presence of multi-scale or hierarchic structure. The hierarchic concept of sea-ice morphology was put forward by Overland et al. (1995). They pointed out the existence of a set of scale levels related to specific formations, such as crystals, blocks, floes, and the basin as a whole. In the framework of the hierarchic approach, the fracture processes that take place on different levels are, to a significant extent, independent (as controlled by forcing of different nature) but they are self-similar. The degree of disconnection of events constitutes the organization of the hierarchic system. The damages of a lower scale level could form a "cluster" of damages that would be an isolated (but structurally similar) damage of a higher level. The inter-level transition in a multi-scale sys- tem can disturb a time sequence of events at adjacent levels if the "reservoir of weak points" on the lower level is depleted, and the number of newly-forming damages of higher level is insufficient for statistics. However, the force distribution remains all the time scaled and unchanged since it does not depend on the events' sequence.

Consequently, the observed response of the temporal statistics of the sea-ice drift to the large-scale perturbation in combination with the invariability of the force distribution can be interpreted as a manifestation of the transient process in the critical, hierarchic system.

The feedback mechanism needed for maintaining the critical state is realized through ice shearings under the action of both external and internal forces. A spectrum of such shearings is related with different modes of deformation and fracture of contact zones in the sea-ice. The horizontal ice shearings result in auto-oscillations of different spatial and temporal scales, which provide the local stress release; the whole system returns to the equilibrium/critical state until another event occur. Thus, the spectrum of cyclic shearings of ice reflects a variety of self-similar processes in the permanently critical system. The parametric interactions of oscillations provide conditions for the energy re-distribution over 
vast areas, what is the requirement for self-organizing of the system.

\section{Conclusions}

The temporal invariance of the ice drift dynamics in combination with the fractal geometry of ice sheets allows one to consider the ASIC as a space-time fractal domain. Large-scale perturbations in the ASIC, which manifest themselves by the short-time basin-wide fragmentations of the ice cover, do not affect neither scaling properties of the fracture pattern, nor self-similarity of the force distribution in the pack; however, the temporal correlation of the ice-field motion becomes disturbed in the periods of fragmentation. This kind of decorrelation is similar to the phase transition in the heterogeneous system whose transient metastable state is characterized by disordered sequence of events. The mechanism of the long-range correlation in the sea-ice cover is related with the self-organized oscillation dynamics inherent in the ice pack.

Edited by: K. Döös

\section{References}

Arneodo, A., Bacry, E., and Muzy, J. F.: The thermodynamics of fractals revisited with wavelets, Physica A, 213, 232-275, 1995.

Bouchaud, E.: Scaling properties of cracks, J. Phys.: Cond. Matter, 9, 4319-4344, 1997.

Caldarelli, G., Di Tolla, F. D., and Petri, A.: Self-organization and annealed disorder in a fracturing process, Phys. Rev. Lett., 77, 2503-2506, 1996.

Carpinteri, A., Lacidogna., G., and Pugno, N.: Scaling of energy dissipation in crushing and fragmentation: a fractal and statistical analysis based on particle size distribution, Int. J. Fracture, 129(1), 131-139, 2004.

Chmel, A. and Smirnov, V. N.: Forestalling response of the correlated sea-ice drift dynamics to the approaching atmospheric depression, Fifth Annual Meeting of the European Meteorological Society, Utrecht, Abstract A-00131, 2005.

Chmel, A., Smirnov, V. N., and Astakhov, A. P.: The fractality of sea ice drift dynamics as revealed from the "North Pole 32" monitoring, JSTAT P02002, 2005.
Christensen, K. and Olami, Z.: Scaling, phase transitions, and nonuniversality in a self-organized critical cellular-automation model, Phys. Rev. A, 46, 1829-1838, 1992.

Hopkins, M. A., Frankenstein, S. F., and Thorndike, A. S.: Formation of an aggregate structure in the Arctic ice pack, J. Geophys. Res., 109, C01032, doi:10.1029/2003JCool1855, 2004.

Kapiris, P. G., Balasis, G. T., Kopabas, J. A., Antonopoulos, G. N., Peratzakis, A. S., and Eftaxias, K. A.: Scaling properties of multiple fracturing of solid materials, Nonlin. Processes Geophys., 11, 137-151, 2004, http://www.nonlin-processes-geophys.net/11/137/2004/.

Kapiris, P. G., Eftaxias, K. A., Nomikos, K. D., Polygiannakis, J., Dologlou, E., Balasis, G. T., Bogris, N. G., Peratzakis, A. S., and Hadjicontis, V. E.: Evolving towards a critical point: A possible electromagnetic way in which the critical regime is reached as the rupture approaches, Nonlin. Processes Geophys., 10, 511-524, 2003, http://www.nonlin-processes-geophys.net/10/511/2003/.

Korsnes, R., Souza, S. R., Donangelo, R., Hansen, A., Paczuski, M., and Sneppen, K.: Scaling in fracture and refreezing of sea ice, Physica A, 331, 291-296, 2004.

Lewis, J. K., Tucker, W. B., and Stein, P. J.: Observation and modeling of thermally induced stresses in first-year ice, J. Geophys. Res., C99, 16361-16371, 1994.

Mandelbrot, B. B., Passoja, D. F., and Paullay, A. J.: Fractal character of fracture surfaces of metals, Nature, 308, 721-723, 1984.

Marsan, D., Stern, H., Lindsay, R., and Weiss, J.: Scale dependence and localization of the deformation of Arctic sea ice, Phys. Rev. Lett., 93, 178501, doi:10.1103/PhysRevLett.93178501, 2004.

Martin, S. and Drucer, R.: Observation of short-period ice floe accelerations during leg II of the Polarbjorn drift, J. Geophys. Res., 96, 10 567-10580, 1991.

Matsushita, M. L.: Fractal viewpoint of fracture and accretion, J. Phys. Soc. Japan, 54, 857-860, 1985.

Overland, J. E., Walter, B. A., Curtin, T. B., and Turet, P.: Hierarchy and sea ice mechanics: a case study from the Beaufort Sea, J. Geophys. Res., 100, 4559-4571, 1995.

Richter-Menge, J. A. and Elder, B. C.: Characteristics of pack ice stress in the Alaskan Beaufort sea, J. Geophys. Res., C103, $21817-21829,1998$.

Smirnov, V. N.: Dynamic processes in sea ice, edited by: Timokhov, L. A., Gidrometeoizdat, St. Petersburg (in Russian), 1995.

Weiss, J.: Scaling of fracture and faulting of ice on earth, Surv. Geophys. 24, 185-227, 2003.

Zang, A., Wagner, F. C., Stanchits, S., Dresen, G., Andresen, R., and Haidekker, M. A.: Source analysis of acoustic emission in Aue granite cores under symmetric and asymmetric compressive loads, Geophys. J. Intern., 135, 1113-1130, 1998. 